\title{
FOXQ1 regulates senescence-associated inflammation via activation of SIRT1 expression
}

\author{
Pan Wang ${ }^{1,2,5}$, Cuicui Lv ${ }^{3,5}$, Tao Zhang ${ }^{4}$, Junling Liu' ${ }^{1}$, Jin Yang ${ }^{1}$, Fangxia Guan ${ }^{\star, 4}$ and Tianpei Hong,
}

Cellular senescence is an initial barrier to tumor development that prevents the proliferation of premalignant cells. However, some of the features of senescent cells seem to promote tumor progression via senescence-associated secretory phenotype (SASP). Here, we demonstrated that the protein level of forkhead box Q1 (FOXQ1), which highly overexpresses in several kinds of tumors, was significantly downregulated during both replicative and oncogene-induced senescence. Moreover, overexpression of FOXQ1 delayed senescence, whereas FOXQ1 silence led to premature senescence in human fibroblasts. Furthermore, we identified that FOXQ1 upregulated SIRT1 expression through transcriptional regulation via directly binding to the SIRT1 promoter. Finally, we showed that FOXQ1 remarkably inhibited the replicative senescence through depressing the expression of the inflammatory cytokines interleukin-6 (IL-6) and IL-8 via modulation of SIRT1-NF- $\kappa$ B pathway. In addition, FOXQ1 overexpressed in human esophageal cancer cells and ablation of FOXQ1 restrained the tumourigenic ability of the esophageal cancer cells (EC109 and EC9706) in a mouse xenograft model in vivo. Taken together, these findings uncover a previously unidentified role of FOXQ1 regulating SASP and tumor development at same time.

Cell Death and Disease (2017) 8, e2946; doi:10.1038/cddis.2017.340; published online 20 July 2017

Cell senescence is a cell cycle arrest program characterized by an irreversible growth arrest in response to diverse forms of cellular stress which renders senescent cells insensitive to mitogenic stimuli and undoubtedly contributes to key senescence phenotypes. ${ }^{1,2}$ Two pathways, p53/p21 and p16 ${ }^{\text {INK4a/ }}$ $\mathrm{Rb}$ (retinoblastoma), can orchestrate the development of senescence in response to DNA damage, oxidative stress and oncogene activation. ${ }^{3}$ The senescence response is widely recognized as a potent tumor suppressive mechanism. ${ }^{4}$ However, recent evidence strengthens the idea that senescent cells seem to mediate the development of age-related pathologies, including cancer. ${ }^{5,6}$ There is mounting evidence that senescent cells, which often persist in vivo, can promote tumor progression in addition to other age-related pathologies via the senescence-associated secretory phenotype (SASP). ${ }^{7,8}$

Senescent cells can secret numerous kinds of pro-inflammatory cytokines, chemokines, growth factors and proteases, which are termed as SASP. ${ }^{9}$ SASP contributes to multiple biological effects depending upon the context. Some SASP factors such as interleukin-6 (IL-6) and IL-8 have been described to reinforce the senescence program in an autocrine manner, and to promote senescence induction in a paracrine mode. ${ }^{9,10}$ Besides, SASP factors can also promote the immune surveillance of senescent cells by stimulating the immune system to clear premalignant senescent cells and facilitate tissue repair. ${ }^{11,12}$ Nevertheless, SASP may promote inflammation and exert a pro-proliferative activity, thus contributing to cancer initiation and progression. ${ }^{13}$ SASP is regulated mainly at $m R N A$ level and depends on NF- $\kappa \mathrm{B}$ and $\mathrm{C} /$ EBP $\beta$ activities. ${ }^{14}$ More recently, it has been shown that IL-1a and NF- $k \mathrm{~B}$ comprise a positive feedback loop that stimulates the transcription of SASP factors. ${ }^{15}$ Additionally, miR-146 a/b, Klotho, protein kinase D1 and GATA4 have also been implicated as a regulator of SASP. ${ }^{16-19}$ Overall, though great progresses have been made in investigating the molecular mechanisms of SASP, there is still a long way to go to illuminate the role of SASP in aging process.

Forkhead box (FOX) family proteins, which characterized by presence of an evolutionarily conserved DNA binding domain, involve in a wide range of crucial cellular processes including stress resistance, metabolism, cell cycle arrest, aging and apoptosis. ${ }^{20}$ Foxq1 was first found in modulating hair follicle development in mammals, ${ }^{21}$ and was also noticed in regulating the major stomach mucin expression and granule content in stomach surface mucous cells in rodents. ${ }^{22}$ Thereafter, evidence indicated that FOXQ1 highly expressed in colorectal cancer, breast cancer, liver cancer, esophageal cancer, nonsmall cell lung cancer and other cancers. ${ }^{23-29}$ It has been reported that miR-320 can suppress colorectal cancer by targeting FOXQ1. ${ }^{30}$ Moreover, FOXQ1 can promote hepatocellular carcinoma metastasis via activating the transcription of ZEB2 and versican V1. ${ }^{31}$ However, its role in longevity remains to be elucidated.

In the present study, we demonstrated that FOXQ1 expression level was downregulated during both replicative

\footnotetext{
${ }^{1}$ Department of Endocrinology and Metabolism, Peking University Third Hospital, Beijing, China; ${ }^{2}$ Department of Clinical Laboratory, Key Clinical Laboratory of Henan Province, The First Affiliated Hospital of Zhengzhou University, Zhengzhou, China; ${ }^{3}$ Department of Clinical Laboratory, The Second Affiliated Hospital of School of Medicine, Zhejiang University, Hangzhou, China and ${ }^{4}$ School of Life Sciences, Zhengzhou University, Zhengzhou, China

${ }^{*}$ Corresponding author: F Guan, School of Life Sciences, Zhengzhou University, Zhengzhou 450001, China. Tel/Fax: + 86371 66913700; E-mail: guanfangxia@ 126.com or T Hong, Department of Endocrinology and Metabolism, Peking University Third Hospital, 49 North Garden Road, Haidian District, Beijing 100191, China. Tel: +86 10 82266918; Fax: +86 10822655 96; E-mail: tpho66@ @jmu.edu.cn

${ }^{5}$ These authors contributed equally to this work.

Received 11.4.17; revised 10.6.17; accepted 13.6.17; Edited by B Zhivotovsky
} 
and oncogene-induced senescence. We characterized that in human 2BS diploid fibroblasts overexpression of FOXQ1 could promote cell growth, while FOXQ1 silencing lead to premature senescence. We also identified that FOXQ1 could downregulate IL-6 and IL-8 expression through SIRT1mediated inhibition of inflammatory pathway. Moreover, we showed that FOXQ1 protein level was elevated in human esophageal cancer cells and the tumourigenic ability of FOXQ1 in the cancer cells was confirmed in a mouse xenograft model in vivo. Our findings may allow more effective approaches to the cancer treatment by inhibiting the expression of FOXQ1 and inducing cell senescence.

\section{Results}

Expression of FOXQ1 is decreased during replicative senescence and oncogene-induced senescence. In view of the positive effect of FOXQ1 on the proliferation and metastasis of cancer cells, we speculated that FOXQ1 might also be involved in cellular senescence. Therefore, we first evaluated FOXQ1 expression in the young and senescent human 2BS fibroblasts and in the Ras oncogene-induced premature IMR90 cells. Western blot results showed that the protein level of FOXQ1 was lower in the senescent 2BS cells than that in the young cells. In contrast, the protein level of $\mathrm{p} 16^{\mathrm{INK} 4 \mathrm{a}}$ was higher in the senescent cells than that in the young cells (Figure 1a). Likewise, quantitative reverse transcription polymerase chain reaction (qRT-PCR) analysis demonstrated downregulation of FOXQ1 and upregulation of $p 16^{\text {INK4a }}$ transcripts in the aged fibroblasts (Figure $1 \mathrm{~b}$ ). To consolidate this observation, we also assessed their protein levels in human IMR90 diploid fibroblasts that were stably integrated with a tamoxifen-regulated form of activated Ras. In line with the above findings in the senescent 2BS cells, we observed a marked reduction of FOXQ1 protein level accompanied by an increased $\mathrm{p} 16^{\mathrm{INK} 4 \mathrm{a}}$ protein level after exposed to 4-hydroxytamoxifen in a time-dependent manner (Figure 1c).

In order to clarify whether the expression profiles of FOXQ1 and $\mathrm{p} 16^{\mathrm{INK} 4 \mathrm{a}}$ with aging could be recapitulated in vivo, we examined their protein level in several tissues from young (3 months) and old (18 months) adult BALB/c mice. The results showed that there was a significant decrease of FOXQ1 level and increase of $\mathrm{p} 16^{\mathrm{INK} 4 a}$ level with age in kidney, brain, spleen and liver (Figure 1d). Collectively, this cell passage- or animal age-dependent reduction of FOXQ1 level suggested that FOXQ1 might be involved in the process of cellular senescence.

FOXQ1 overexpression delays cellular senescence, whereas FOXQ1 silencing leads to premature senescence in human fibroblasts. To determine the functional role of FOXQ1 in the cell senescence, FOXQ1 was overexpressed and silenced, respectively, with a retrovirus expression system in the 2BS cells. Cell proliferation and senescence markers were then monitored at several time points. Growth curve and crystal violet staining assays indicated that the 2BS cells with ectopic FOXQ1 expression displayed higher proliferation rate (Figure 2a) and more colony formation (Figure $2 \mathrm{~b}$ ) than those in the cells with empty vector. Next, we took a short hairpin RNA (shRNA)based knockdown approach to examine the requirement of FOXQ1 for senescence progression. In concert with the effect of ectopic FOXQ1 expression, its removal resulted in lower proliferation rate (Figure 2a) and less colony formation (Figure 2b) than those in the cells transduced with empty vector. Meanwhile, miR30-FOXQ1 also resulted in emerging the morphological features of senescence, characterized by enlarged and flattened cell size, increased senescenceassociated heterochromatin foci (Figure 2c), elevated activity of senescence-associated $\beta$-galactosidase (SA- $\beta$-gal), a biomarker for senescent cells (Figures $2 \mathrm{~d}$ and $\mathrm{e}$ ), and reduced $S$ and increased $\mathrm{G} 1$ compartments (Figure 2f) compared with scramble control vector. Conversely, LPC-FOXQ1 induced much lower SA- $\beta$-gal activity (Figures $2 \mathrm{~d}$ and $\mathrm{e}$ ) and less cell cycle progression (Figure 2f) than the infection with its corresponding empty control vector. These results demonstrated that FOXQ1 was essential for replicative cell senescence and that FOXQ1 overexpression promoted human fibroblast proliferation, whereas FOXQ1 silencing induced the cell senescence.

FOXQ1 increases the mRNA and protein levels of SIRT1. As mentioned above, our results showed that FOXQ1 overexpression promoted cell growth, whereas FOXQ1 knockdown led to growth inhibition. We therefore examined the molecular mechanism by which FOXQ1 delayed cellular senescence. As SIRT1 is an important determinant of longevity that regulates lifespan in diverse species, ${ }^{32}$ and mounting evidences support the concept that SIRT1 is an essential regulator of inflammation by altering histones and transcription factors such as $\mathrm{NF}-k \mathrm{~B},{ }^{33}$ we thus investigated whether FOXQ1 could mediate cell senescence via directly regulating SIRT1. Western blot analysis revealed that FOXQ1 overexpression significantly increased the protein level of SIRT1 in HEK293T cells (Figure 3a). In addition, we silenced the FOXQ1 gene and examined SIRT1 protein levels by western blot. As expected, we noticed that a markedly decreased level of SIRT1 protein in the FOXQ1 siRNAtransfected cells compared with the control scrambled siRNA-transfected cells (Figure 3a). Meanwhile, the protein levels of p65RelA and $I_{\kappa} \mathrm{B} a$ were also analyzed by western blot. We found that the protein level of $I_{\kappa} \mathrm{B} a$, an inhibitor of NF$\kappa \mathrm{B}$, positively correlated with FOXQ1 and SIRT1, while the level of p65RelA displayed an opposite tendency with FOXQ1 and SIRT1 (Figure 3a). To further strengthen this argument, we overexpressed FOXQ1 by pLPC-Puro retroviral vector and silenced it using miR30 retroviral vector in the 2BS cells. We observed the identical results to those from HKE293 cells (Figure 3b). Besides, increased FOXQ1 expression resulted in a decreased level of $\mathrm{p} 16^{\mathrm{INK} 4 \mathrm{a}}$ protein, while removal of FOXQ1 exhibited an opposite effect on p16 ${ }^{\text {INK4a }}$ protein level (Figure $3 b$ ). In parallel with the findings from western blot analysis, qRT-PCR analysis also showed a positive regulation of SIRT1 mRNA expression by FOXQ1 (Figures $3 \mathrm{c}$ and $\mathrm{d}$ ), indicating that FOXQ1 could activate transcription of SIRT1. 
a

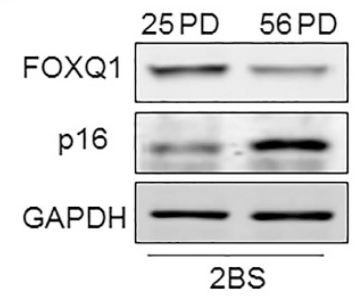

C

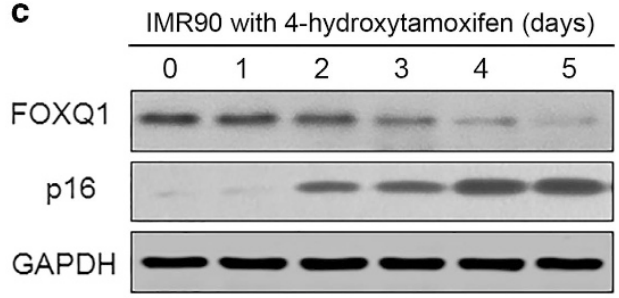

d

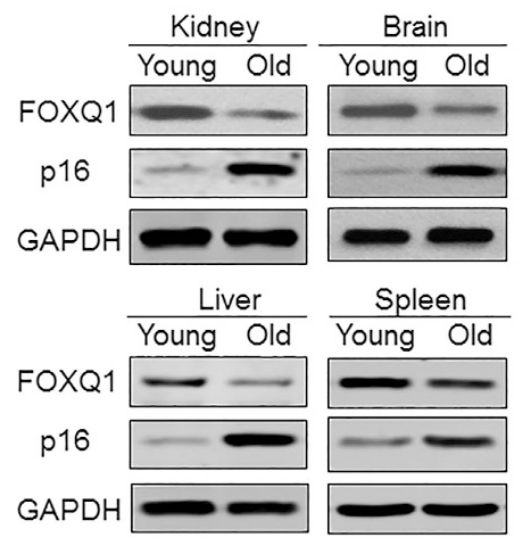

Figure 1 Expression of FOXQ1 decreases with senescence. (a) Western blot analysis of FOXQ1 and p16 $6^{\text {INK4a }}$ level in 25 PD and 56 PD 2BS cells. (b) qRT-PCR analysis of FOXQ1 and $p 16^{I N K 4 a}$ in the young and old 2BS cells. The mRNA expression levels of indicated gene were normalized to $\beta$-actin. Each bar represents the means \pm S.D. for triplicate experiments. ${ }^{*} P<0.05$. (c) FOXQ1 was time-dependently downregulated in IMR90 cells with tamoxifen-induced Ras expression. Protein level of FOXQ1 and p16 ${ }^{\text {INK4a }}$ at different times following 4-hydroxytamoxifen treatment was determined by western blot analysis. (d) Protein levels of FOXQ1 in indicated tissues of young ( 3 months) and old (18 months) BALB/c mice were determined by western blot analysis. GAPDH was used as loading control

FOXQ1 promotes proliferation through suppressing IL-6 and IL-8 expression via transcriptional upregulation of SIRT1. It is well known that SIRT1 is a key regulator of inflammation in mammalian cell via inhibition of NF- $k \mathrm{~B}$ activity. Based on our above observation that FOXQ1 could increase the protein levels of both SIRT1 and $I_{k} \mathrm{~B} a$, we prudently inferred that FOXQ1 might regulate cell senescence via modulating senescence-associated inflammation. To further substantiate the inference, we performed experiments with gain- and loss-of-function of FOXQ1 in the 2BS cells. As IL-6 and IL-8 are both required for the inflammatory network and senescence entry and maintenance, we tested whether FOXQ1 could regulate IL-6 and IL-8 expression via modulating SIRT1 activity. Here we used qRT-PCR to determine the mRNA levels of IL-6 and IL-8. LPC-FOXQ1 markedly decreased the mRNA abundance of $I L-6$ and $I L-8$ compared with control transfection (Figure 4a). In contrast, FOXQ1 deletion significantly elevated IL-6 and IL-8 mRNA levels (Figure 4b). Moreover, the secreted levels of IL-8 in supernatant detected by enzyme linked immunosorbent assay (ELISA) showed similar alteration with its mRNA level (Figure 4c). All together, these results indicated that FOXQ1 was an important mediator in SASP by regulating IL-6 and IL-8 induction.

We next sought to explore the molecular mechanism underlying the FOXQ1-mediated transcriptional activation of SIRT1. To test the possibility that FOXQ1 directly bound to the SIRT1 promoter region, we performed a chromatin immunoprecipitation (ChIP) assay followed by quantitative PCR (ChIP-qPCR). The ChIP-qPCR results indicated a significant enrichment of FOXQ1 in the promoter of SIRT1 in the FOXQ1 overexpressed cells compared with the control cells (Figure 4d). To investigate the functional importance of the FOXQ1-SIRT1 axis in SASP, we used a pharmacological method to clarify whether the regulation of IL-6 and IL-8 expression by FOXQ1 was dependent on SIRT1 activity. We performed an epistasis analysis by overexpression of FOXQ1 with or without EX-527, a specific SIRT1 inhibitor. Western blot results revealed that the FOXQ1-induced upregulation of $I_{\kappa} \mathrm{B} a$ level was abolished by addition of EX-527 treatment (Figure 4e). In addition, real-time PCR results demonstrated that FOXQ1 overexpression led to the decrease of $I L-6$ and IL-8 expression levels, which was eliminated by inhibition of SIRT1 activity with EX-527 (Figure 4f). These results suggested that the FOXQ1-mediated regulation of SASP factors was dependent on SIRT1-NF-kB pathway.

FOXQ1 highly expressed in esophageal cancer cells contributes to cell proliferation in vitro. It is well known that cellular senescence is a potent tumor suppressive mechanism characterized by an irreversible growth arrest. However, several lines of evidence show that senescent cells can also promote tumor progression via SASP. ${ }^{7,13}$ In the present study, we showed that FOXQ1 could promote cell proliferation and delay cell senescence by inhibition of IL- 6 and IL-8 expression. Therefore, we curiously wanted to know the expression profile of FOXQ1 in cancer cells and its role in the cancer development. To this end, we first measured FOXQ1 protein level in 5 human esophageal cancer cell lines (EC1, EC109, EC9706, TE1 and TE13) and the normal 


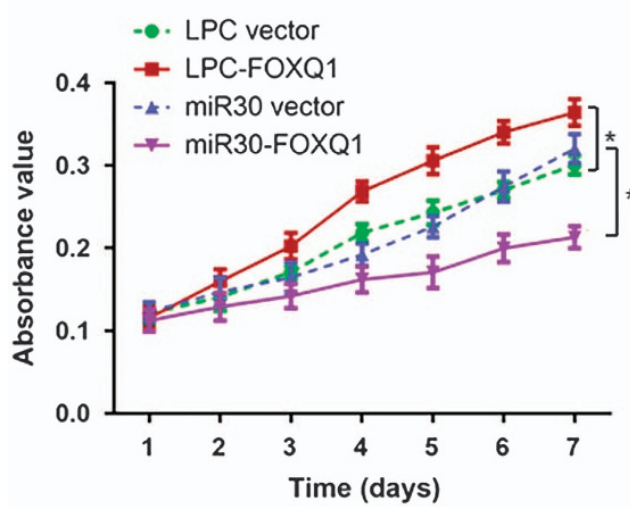

b
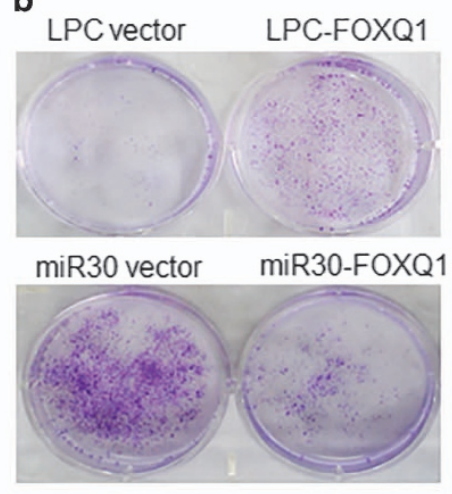

c
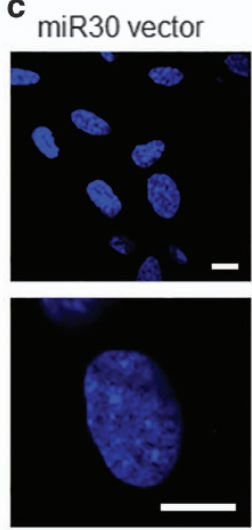
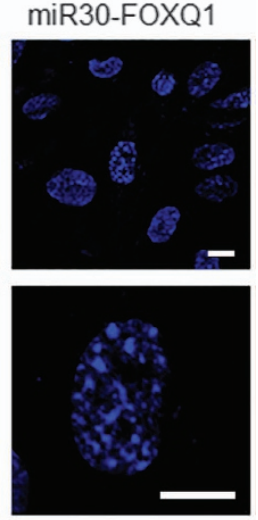

d

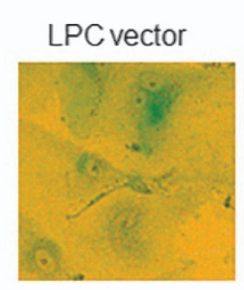

miR30 vector

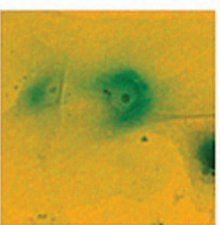

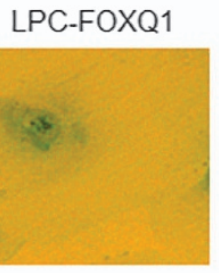
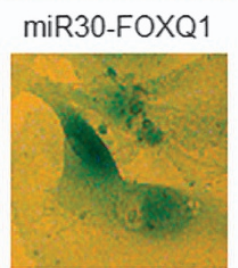
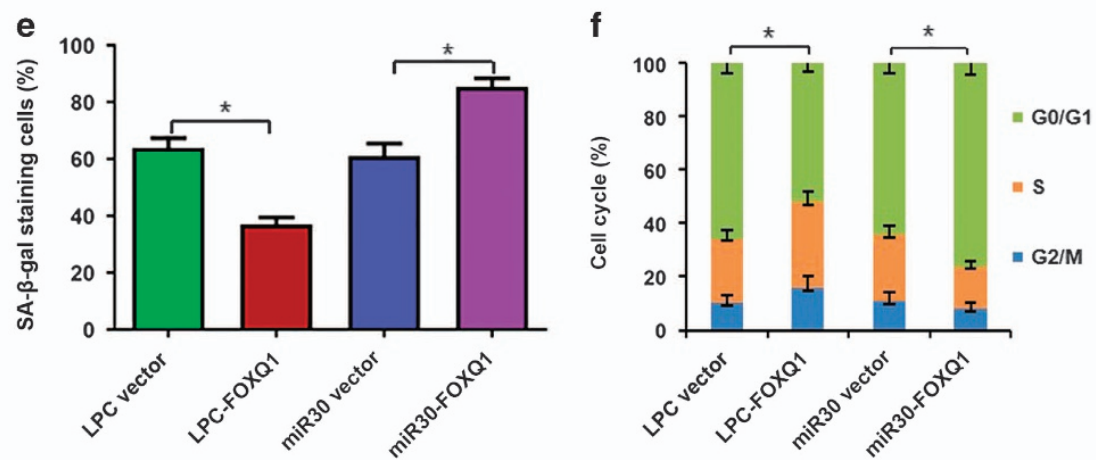

Figure 2 FOXQ1 overexpression promotes cell proliferation and FOXQ1 silence causes premature senescence. (a) The 2BS cells expressing the indicated genes and shRNAs were cultured and growth curves were determined by the MTT assay. (b) The 2BS cells were infected with indicated retroviruses and cultured in the 6-well plates for 6 days, followed by fixation and staining with crystal violet. (c and $\mathbf{d})$ Representative images of the indicated cells with stained for senescence-associated heterochromatin foci by DAPI (c) and SA- $\beta$-gal activity (d) were shown (Scale bar, $10 \mu \mathrm{m}$ ). (e) The percentage of cells positive for SA- $\beta$-gal in each sample was determined. At least 300 cells were counted for each sample. (f) Cell cycle was measured. Values are means \pm S.D. of triplicate points from a representative experiment, which was repeated three times with similar results. ${ }^{*} P<0.05$

human esophageal epithelial cell line HET-1A. The relative level of FOXQ1 protein was higher in all the esophageal cancer cell lines than that of the HET-1A cells (Figure 5a). Next, we silenced FOXQ1 by using lentivirus in EC109 and EC9706 cells. The MTT assay demonstrated that silence of FOXQ1 caused cell growth retardation compared with its corresponding empty vector control (Figure 5b). These findings indicated that FOXQ1 might contribute to the progression of esophageal cancers, which is in agreement with the results of previous studies. ${ }^{28}$

FOXQ1 affects tumor growth in a mouse xenograft implantation model. Given that our in vitro studies suggested a functional role for FOXQ1 in the esophageal cancer cell proliferation, we next investigated the contribution of FOXQ1 to the esophageal cancer cell growth in vivo. To this end, stable EC109 and EC9706 cells transduced with either FOXQ1 silencing or control lentiviral vector were subcutaneously injected into the flank of NOD/SCID mice, and tumor growth was monitored over a period of 30 days. The mean tumor volume and weight of the FOXQ1 silencing vector- expressing EC109 and EC9706 xenografts grew at a slower rate than those derived from the xenografts expressing control vector (Figures $6 \mathrm{a}-\mathrm{c}$ ). Taken together, these results suggested that FOXQ1 might promote cell proliferation and tumourigenicity of the esophageal cancer cells in vivo.

\section{Discussion}

It has been reported that cellular senescence functions as a general protective mechanism against proliferative stress responses and cancer development in vivo early in life, however, late in life senescent cells accumulate in different tissues in aging mammals and seem to mediate the development of age-related pathologies, including cancer. ${ }^{5}$ FOXQ1 has been found to regulate a number of processes including cell proliferation, differentiation and development, especially tumor proliferation. ${ }^{21-29}$ In this study, we showed that the protein level of FOXQ1 was decreased during cell senescence both in vitro and in vivo. Moreover, FOXQ1 overexpression delayed cellular senescence, whereas FOXQ1 silencing led to premature senescence in human fibroblasts. These results suggest that FOXQ1 is involved in the regulation of cellular 
a

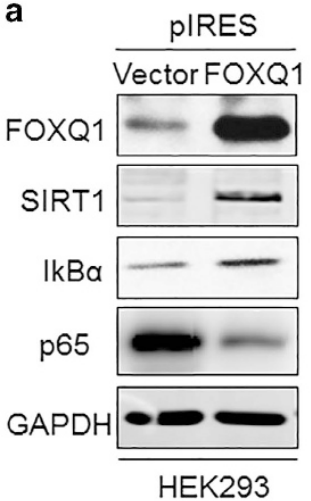

b

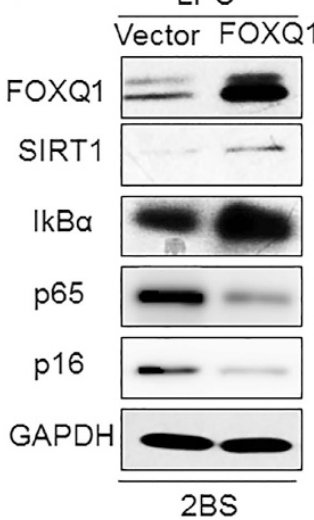

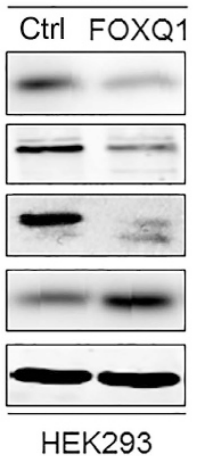

$\operatorname{miR} 30$
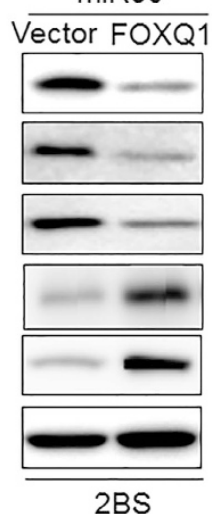

C

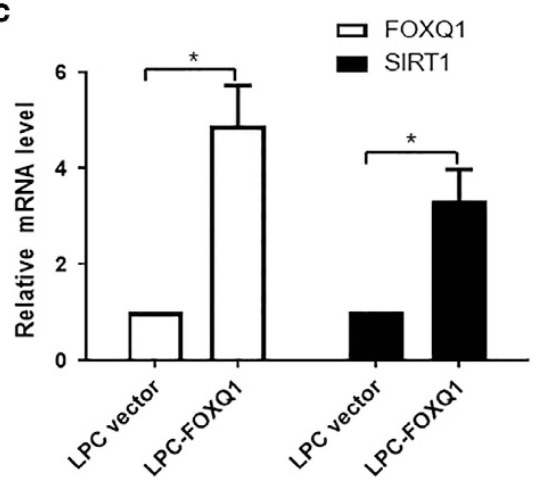

d

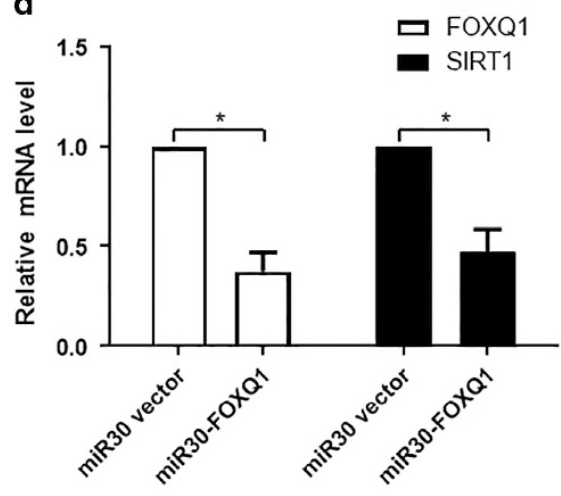

Figure 3 FOXQ1 upregulates SIRT1 at mRNA and protein levels. (a) Western blot analysis of FOXQ1, SIRT1, IkB $\alpha$ and p65 level was carried out in FOXQ1 overexpression or knockdown cells compared with control cells. HEK293 cell was used for these experiments. (b) The 2BS cells were stably transduced LPC-FOXQ1, miR30-FOXQ1 or control vector with retrovirus. The infected 2BS cells were selected and then the indicated proteins were determined by western blot. (c and d) The mRNA expression level of FOXQ1 and SIRT1 were determined qRT-PCR. Data represent the means \pm S.D. for triplicate experiments. ${ }^{*} P<0.05$

senescence. To our knowledge, this is the first report that FOXQ1 can regulate cell senescence except tumor development and metastasis. Most importantly, we deciphered that FOXQ1 modulated cell senescence via positively regulating the NAD-dependent deacetylase SIRT1 at transcriptional level. Given the definite role of SIRT1 in cell senescence, our data identified the undisputed importance of FOXQ1SIRT1 signaling in cell senescence.

Chronic inflammation has been reported to be the reason of many age-related diseases, including atherosclerosis, heart failure, osteoporosis, neurodegeneration and cancer. ${ }^{6}$ SASP is characterized by being an indispensable constituent of chronic inflammation and is proposed to have important roles in aging and many senile diseases. ${ }^{13}$ However, the causes and consequences of SASP are not very clear. By gain- and loss-of-function studies, we demonstrated that FOXQ1 overexpression could suppress the expression of some SASP factors such as IL-6 and IL-8, which might account for the effect that FOXQ1 was able to delay cell senescence in the human 2BS fibroblasts. Conversely, FOXQ1 silencing could stimulate the production of these SASP factors and might thus contribute to the premature senescence. Therefore, we deciphered a previously unidentified role and mechanism of SASP in cell senescence for the first time.

In the present study, we also noticed that FOXQ1 highly expressed in all the 5 human esophageal cancer cell lines.
Compared with the corresponding tumor cells transduced with empty control vector, the growth of human esophageal cancer cells was retarded in the FOXQ1 silencing vector-expressing EC109 and EC9706 cells both in vitro and in vivo. These findings are in agreement with the previous reports that FOXQ1 is involved in the development and progression of malignant tumors including esophageal cancer. ${ }^{23-29}$ Therefore, FOXQ1 might potentially act as an important regulator in the maintenance of the balance between cell senescence and tumor development. The different expression level of FOXQ1 might lead to different cell fate as black and white. Furthermore, it has been reported that Foxq1 is restrictedly expressed in rapidly proliferated stomach cells, ${ }^{22}$ which consolidates its role in promoting cell proliferation once more. On the other hand, the degradation of FOXQ1 protein during cell senescence is considerable and the invalidation of this process might be the reason why high level of FOXQ1 protein is found in several kinds of tumor tissues and can thus promote cancer cell proliferation and metastasis. ${ }^{23-29}$ The mechanism through which FOXQ1 is degraded in cell senescence remain to be identified in the future study. Given that Foxq1 mutant mice are available, ${ }^{22}$ it can be helpful to use this animal model to investigate tissue and organ senescence in our future work.

SASP is regulated mainly by NF- $\kappa \mathrm{B}$ and C/EBP $\beta$, and SIRT1 is a key regulator of inflammation in mammalian cells mainly through deacetylation of $p 65$, thus inhibiting NF-KB 
a

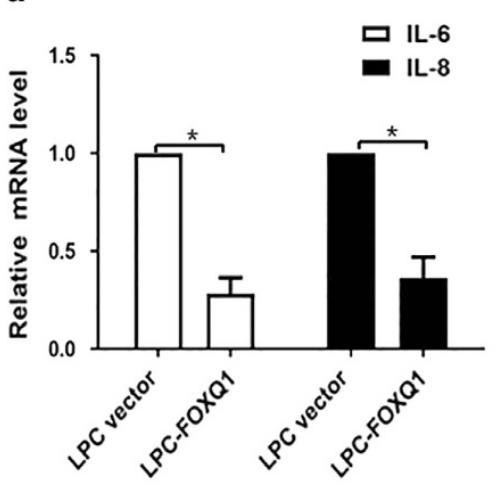

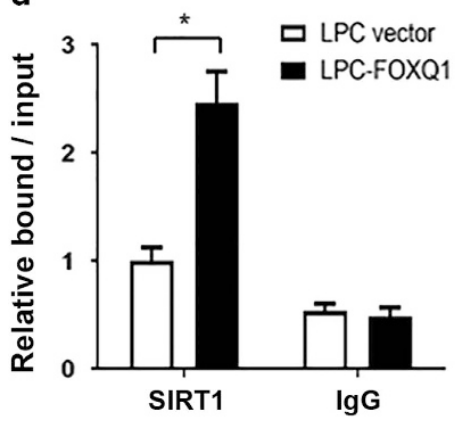

b

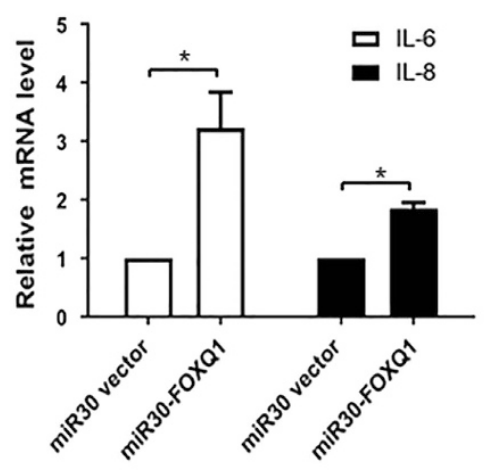

e

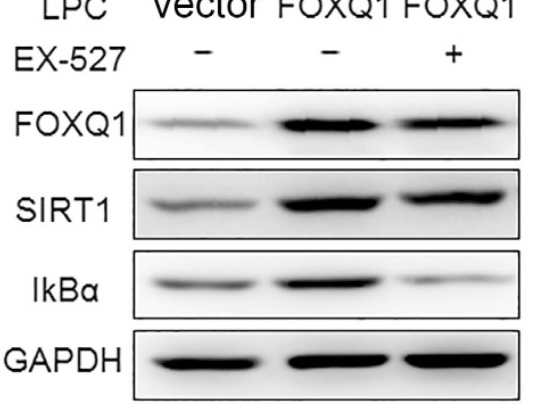

C

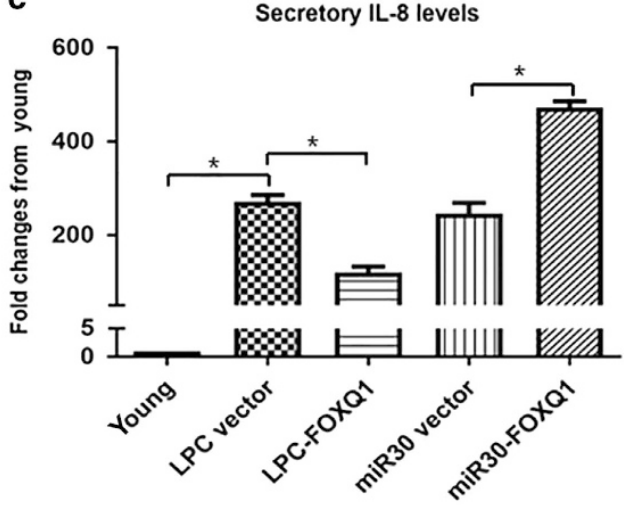

$\begin{array}{ll}\mathbf{1} & \text { LPC vector } \\ 1.5] & \text { LPC-FOXQ1 } \\ & =\text { LPC-FOXQ1+EX-527 }\end{array}$

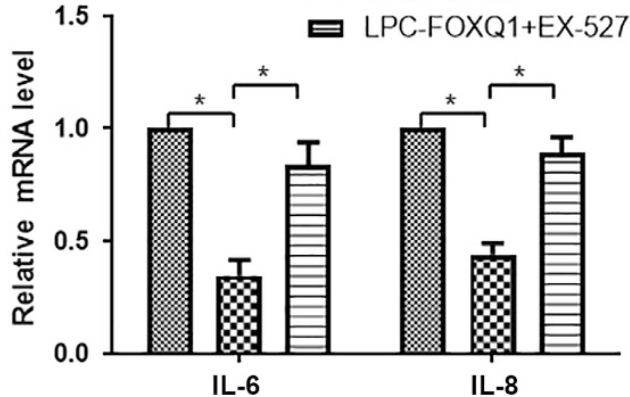

Figure 4 Regulation of IL-6 and IL-8 expression by FOXQ1 is dependent on SIRT1 activity. (a-c) Ectopic expression of FOXQ1 inhibited, and FOXQ1 silencing promoted IL-6 and IL-8 expression. The 2BS cells stably transduced with LPC-FOXQ1 (a) or miR30-FOXQ1 (b) were selected and cultured for three days. Total RNA was extracted from the 2BS cells expressing the indicated genes and the relative mRNA level of IL-6 and IL-8 were determined by quantitative PCR. Supernatants collected from the above cells were used to assess secretory levels of IL-8 by ELISA (c). The IL-8 level was standardized with that of the young cells. (d) FOXQ1 could bind to the promoter of SIRT1. The 2BS cells were stably transduced with the LPC-FOXQ1 and control vectors. After selection, the cell lysates were analyzed by ChIP assay using an antibody against FOXQ1. FOXQ1 binding to the SIRT1 promoters is represented as level relative to mouse IgG binding. (e) SIRT1 inhibitor EX-527 abolishes the FOXQ1-mediated IkB $\alpha$ upregulation. The 2BS cells expressing LPC-FOXQ1 were treated with EX-527 $(10 \mu \mathrm{M})$ for $24 \mathrm{~h}$, and then the cells were harvested and analyzed for the level of indicated proteins. ( $\mathrm{f}$ ) EX-527 eliminated the FOXQ1-induced inhibition of IL-6 and IL-8 transcriptional activity. Total RNA was extracted from the cells as indicated in (e) and the relative mRNA levels of IL-6 and IL-8 were determined by quantitative PCR. Error bars represent means \pm S.D. $n=3 .{ }^{*} P<0.05$

activity. ${ }^{34}$ Here, we found that the level of $I_{\kappa} B a$, an inhibitor of $\mathrm{NF}-\kappa \mathrm{B}$, was positively correlated with SIRT1, which implied that the inhibitory effect of SIRT1 on NF- $\kappa$ B activity was mainly dependent on $\mathrm{I}_{\kappa} \mathrm{B} a$ activity in this study. In fact, our study also showed that the FOXQ1 overexpression-induced upregulation of $\mathrm{I} \kappa \mathrm{B} a$ protein level and downregulation of IL-6 and IL-8 expression were abolished by the specific SIRT1 inhibitor EX-527. These results indicate that SIRT1 may strengthen the inhibitive effect on NF- $\kappa \mathrm{B}-$ mediated inflammatory pathway through promoting $\mathrm{I}_{\kappa} \mathrm{B} a$ expression in addition to deacetylation of p65.

In summary, this study demonstrated a previously unknown role of FOXQ1 in cell senescence. Overexpression of FOXQ1 promoted fibroblast proliferation and upregulated the level of SIRT1 expression. Besides, decreased level of FOXQ1 expression during cell senescence induced IL-6 and IL-8 expression, and subsequently reinforced cell senescence via transcriptional inhibition of SIRT1. In this study, we also identified that FOXQ1 highly expressed in human esophageal cancer cells and that FOXQ1 silence inhibited the tumor cell growth both in vitro and in vivo. Therefore, our study uncovers a role and mechanism of FOXQ1 in the regulation of cell senescence, offering new insights on the balance between cell senescence and tumor development.

\section{Materials and Methods}

Plasmids, antibodies, reagents and animals. Full-length forkhead box Q1 (FOXQ1) was cloned from the CDNA of normal human fibroblast using a PCR-based approach and was subcloned into pLPC-pure vector and pIRES vector respectively. The shRNA plasmid was designed and cloned as described. ${ }^{35}$ The primary antibodies used for western blot analysis were as follows: anti-FOXQ1, anti-SIRT1, anti-p16 and anti- $\beta$-actin were from Santa Cruz Biotechnology (Santa Cruz, CA, USA). Anti-I $\kappa \mathrm{B} \alpha$ and anti-p65 was purchased from Cell Signaling Technology (Beverly, MA, USA). Anti-GAPDH was from Proteintech Group (Wuhan, China). 4-Hydroxytamoxifen (T5648, Sigma, St. Louis, MO, USA) was dissolved in methanol; and EX-527 (Selleck Chemicals, Shanghai, China) was dissolved in DMSO (D2650, Sigma). BALB/c mice, which were obtained from Beijing Vital River Laboratory Animal Technology Co., Ltd. (Beijing, China), were maintained in a certified animal facility in accordance with the guidelines set forth by the Animal Ethics Committee of the First Affiliated Hospital of Zhengzhou University.

Cell lines, cell culture and viral infections. Human diploid 2BS fibroblasts (National Institute of Biological Products, Beijing, China) as well as IMR90 human diploid fibroblasts transduced with an ER:Ras (kindly provided by Dr. Masashi Narita, Cancer Research U.K., Cambridge Research Institute) were cultured in Dulbecco's modified Eagle's medium supplemented with 10\% FBS (HyClone, Logan, UT, USA) and antibiotics. The 2BS cells are considered to be young at $30 \mathrm{PD}$ or less and replicative senescent $2 \mathrm{BS}$ cells are defined as $>55$ 


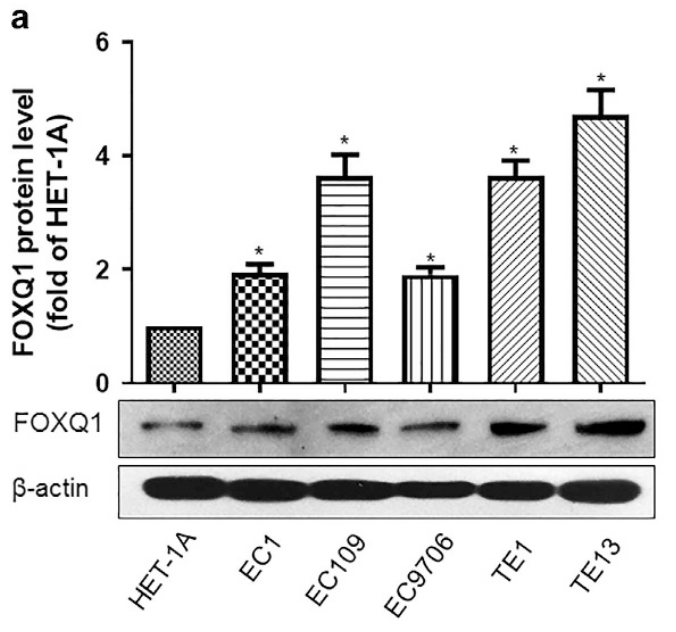

b
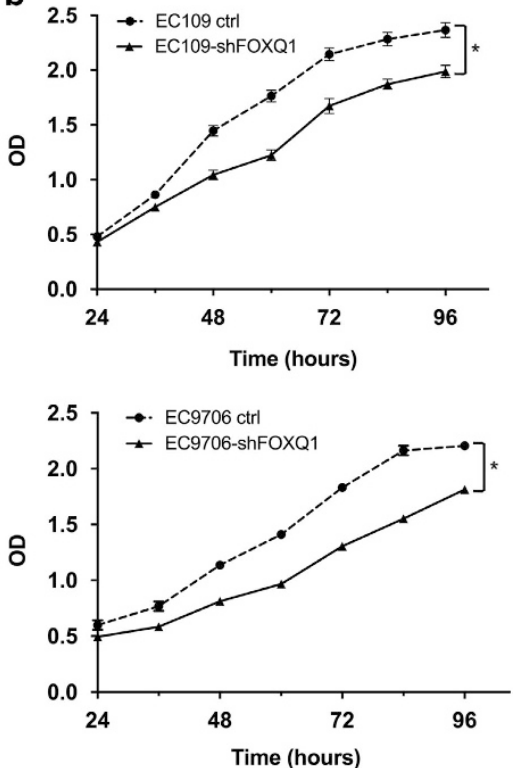

Figure 5 FOXQ1 overexpressed in esophageal cancer cells contributes to tumor cell proliferation in vitro. (a) Western blot analysis of FOXQ1 protein levels in human esophageal cancer cell lines and the human esophageal epithelial cell line HET-1A. The relative level of FOXQ1 protein is normalized to $\beta$-actin (loading control). (b) EC109 and EC9706 cells stably expressing shFOXQ1 or control vector were seeded at the same number in each well. The cell growth was monitored by MTT assay every $12 \mathrm{~h}$. Values are means \pm S.D. of triplicate points from a representative experiment, which was repeated three times with similar results. ${ }^{*} P<0.05$

a

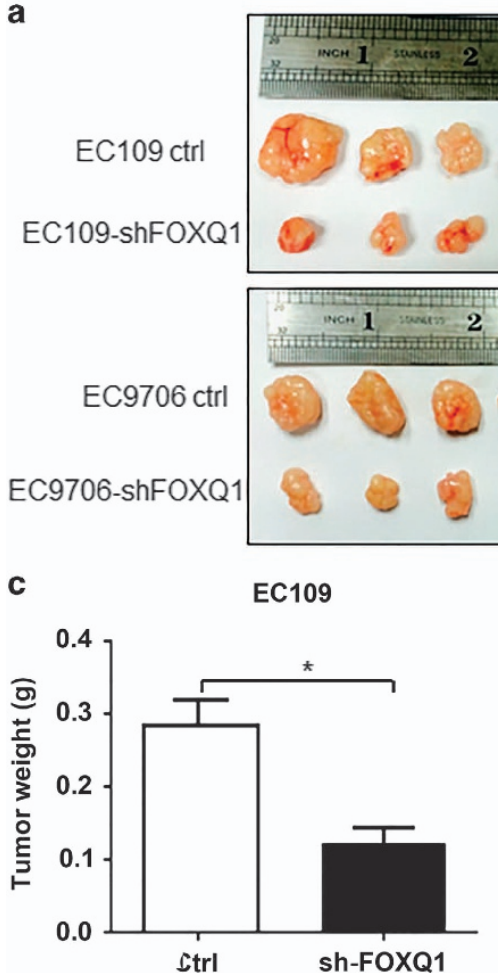

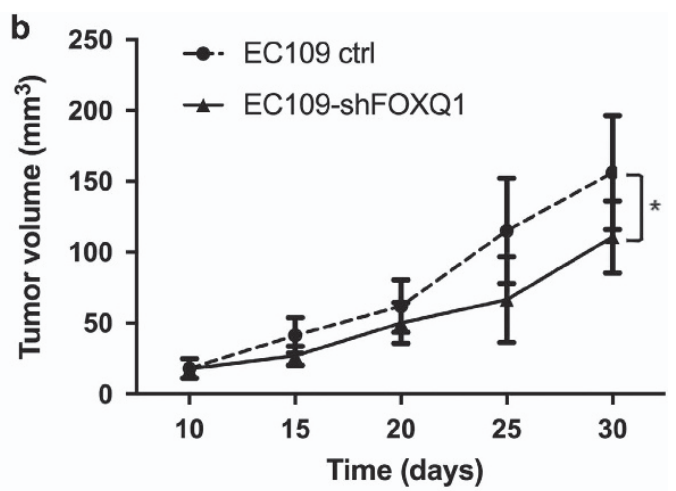

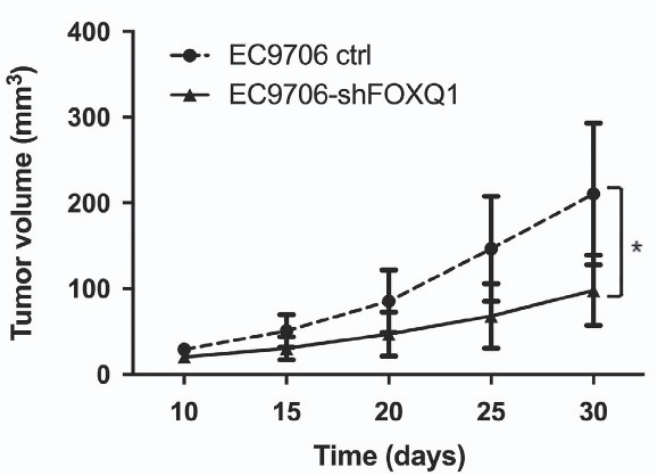

Figure 6 FOXQ1 promotes tumourigenicity in vivo. (a) FOXQ1 silence inhibits the xenograft tumor growth of EC109 and EC9706 cells. The cells $\left(1 \times 10^{6}\right.$ cells) stably expressing FOXQ1 silencing or control lentiviral vector were injected subcutaneously into the left or right flank of NOD/SCID mice, respectively. At 30 days after injection, tumors were excised and photographed. (b) Tumor growth curves in mouse xenograft model. Tumor diameters were measured at the indicated time points, and tumor volumes were calculated. (c) Tumor weights from the same experiment as above on day 30. Values are means \pm S.D. $n=8 .{ }^{\star} P<0.05$ 
PD. To induce senescence, the IMR90 cells expressing ER:H-RasV12 were exposed to $100 \mathrm{nM}$ 4-hydroxytamoxifen. Retroviruses were packed using Phoenix cells and the infections were performed as previously described. ${ }^{36}$ Besides, HET-1A, EC1, EC109, EC9706, TE1 and TE13 cells were maintained in RPMI-1640 medium supplemented with $10 \%$ FBS

RNA interference. Chemically synthesized double-stranded siRNA was used against the transcript of FOXQ1. Cells were transfected with siRNA oligonucleotides for $72 \mathrm{~h}$ using Lipofectamine RNAiMAX (Thermo Fisher Scientific, Waltham, MA, USA).

Quantitative real-time RT-PCR. Total RNA was isolated from the 2BS cells using an RNeasy Mini kit (QIAGEN, Gaithersburg, MD, USA) according to the manufacturer's instructions and then subjected to reverse transcription using the StarScript first strand cDNA synthesis kit (Transgen Biotech, Beijing, China). Real-time PCR was performed in triplicate using the SYBR Green PCR Master Mix (Applied Biosystems, Foster City, CA, USA) on an ABI Prism 7500 sequence detector (Applied Biosystems). The $\beta$-actin gene served as an endogenous control for normalization. The primers were summarized in Supplementary Table 1.

Western blotting. Whole cell extracts were generated by direct lysis with radioimmune precipitation assay (RIPA) buffer (Beyotime, Shanghai, China) with protease inhibitor mixture (Thermo Fisher Scientific). Protein concentration was determined by BCA Protein Assay Reagent (Thermo Fisher Scientific). Samples were boiled by addition $6 \times$ SDS sample buffer for $10 \mathrm{~min}$ at $100{ }^{\circ} \mathrm{C}$ and $40 \mu \mathrm{g}$ of protein was utilized for each western blot. The primary antibodies used for western blot analysis were described as above. The intensity of the protein bands was quantified by densitometry using ImageJ software (NIH, Bethesda, MD, USA). Each western blot was repeated at least three times.

ELISA. The supernatants of the 2BS cells were collected after treatment as indicated. IL-8 level was measured by an ELISA kit from Boster (Wuhan, China).

ChIP assay. ChIP assays were performed using the Chromatin Immunoprecipitation Assay kit (Upstate, New York, NY, USA) according to manufacturer's instruction. DNA released from the precipitated complexes was amplified by realtime PCR using sequence specific primers.

SA- $\beta$-gal analysis. For SA- $\beta$-gal staining, the cells were washed twice with phosphate buffered saline (PBS), fixed for 3-5 min at room temperature in $3 \%$ formaldehyde and washed again with PBS. Cells were then incubated overnight at $37^{\circ} \mathrm{C}$ without $\mathrm{CO}_{2}$ in a freshly prepared staining buffer.

Cell proliferation assay. Growth curves were assayed using the $3-(4,5-$ dimethylthiazol-2-yl)-2,5-diphenyltetrazolium bromide (MTT) method. Cells were seeded into 96-well plates and cultured for periods. At the indicated time, an aliquot of cells were stained with $20 \mu$ l of MTT reagent ( $5 \mathrm{mg} / \mathrm{ml}$ in PBS; M2128, Sigma) for $1 \mathrm{~h}$ and then treated with DMSO for $10 \mathrm{~min}$. The optical density was measured at $570 \mathrm{~nm}$.

Colony formation. In order to determine the colony formation, cells were cultured in plates. Six days later, cells were fixed in $3 \%$ formaldehyde at $37^{\circ} \mathrm{C}$ for $30 \mathrm{~min}$ and washed twice with $1 \times$ PBS, then stained with crystal violet for $1 \mathrm{~h}$ and washed with $1 \times$ PBS twice.

Cell cycle analysis. When cells reached $70-80 \%$ confluence, they were washed with PBS, and then detached with $0.25 \%$ trypsin and fixed with $75 \%$ ethano overnight. Thereafter, the cells were treated with $1 \mathrm{mg} / \mathrm{ml}$ RNase A (R6513, Sigma) at $37^{\circ} \mathrm{C}$ for $30 \mathrm{~min}$, resuspended in $0.5 \mathrm{ml}$ of PBS and stained with propidium iodide in the dark for $30 \mathrm{~min}$. Fluorescence was measured with a FACScan flow cytometry system (BD Biosciences, Lake Franklin, New Jersey, USA).

Tumourigenic assay. EC109 and EC9706 cells were resuspended in $100 \mu$ PBS and $1 \times 10^{6}$ cells were injected subcutaneously into the left or right side of each NOD/SCID mice (Beijing Vital River Laboratory Animal Technology Co., Ltd.). One side was implanted with control tumor cells, and the other with tested tumor cells. Tumor sizes were monitored every five days, and tumor volumes were calculated as volume $=$ length $\times$ width $^{2} \times(1 / 2)$. Animals were maintained on regular food and water. All procedures were approved by the Institutional Animal Care and Use Committee.
Statistical analysis. Data are presented as the means \pm S.D. Statistical difference between groups was analyzed by a Student $t$-test or one-way ANOVA tests where appropriate. Besides, a Bonferroni correction was done when multiple comparisons were performed. A $P$-value $<0.05$ was considered as statistically significant.

\section{Conflict of Interest}

The authors declare no conflict of interest.

Acknowledgements. This work was supported by research grants from the National Natural Sciences Foundation of China (81501200 and 81570692), and China Postdoctoral Science Foundation (2015M572122 and 2017M610025).

\section{PUBLISHER'S NOTE}

Springer Nature remains neutral with regard to jurisdictional claims in published maps and institutional affiliations.

1. Campisi J. Senescent cells, tumor suppression, and organismal aging: good citizens, bad neighbors. Cell 2005; 120: 513-522.

2. Sharpless NE, Sherr CJ. Forging a signature of in vivo senescence. Nat Rev Cancer 2015; 15: $397-408$.

3. Bringold F, Serrano M. Tumor suppressors and oncogenes in cellular senescence. Exp Gerontol 2000; 35: 317-329.

4. Campisi J. Cellular senescence as a tumor-suppressor mechanism. Trends Cell Biol 2001; 11: S27-S31.

5. Campisi J. Aging, cellular senescence, and cancer. Annu Rev Physiol 2013; 75: 685-705.

6. Tchkonia T, Zhu Y, van Deursen J, Campisi J, Kirkland JL. Cellular senescence and the senescent secretory phenotype: therapeutic opportunities. J Clin Invest 2013; 123: 966-972.

7. Coppe JP, Patil CK, Rodier F, Sun Y, Munoz DP, Goldstein J et al. Senescence-associated secretory phenotypes reveal cell-nonautonomous functions of oncogenic RAS and the p53 tumor suppressor. PLOS Biol 2008; 6: 2853-2868.

8. Kuilman T, Peeper DS. Senescence-messaging secretome: SMS-ing cellular stress. Nat Rev Cancer 2009; 9: 81-94

9. Acosta JC, O'Loghlen A, Banito A, Guijarro MV, Augert A, Raguz S et al. Chemokine signaling via the CXCR2 receptor reinforces senescence. Cell 2008; 133: 1006-1018.

10. Kuilman T, Michaloglou C, Vredeveld LC, Douma S, van Doorn R, Desmet CJ et al. Oncogene-induced senescence relayed by an interleukin-dependent inflammatory network. Cell 2008; 133: 1019-1031.

11. Kang TW, Yevsa T, Woller N, Hoenicke L, Wuestefeld T, Dauch D et al. Senescence surveillance of pre-malignant hepatocytes limits liver cancer development. Nature 2011; 479: 547-551.

12. Krizhanovsky V, Yon M, Dickins RA, Hearn S, Simon J, Miething C et al. Senescence of activated stellate cells limits liver fibrosis. Cell 2008; 134: 657-667.

13. Lasry A, Ben-Neriah Y. Senescence-associated inflammatory responses: aging and cancer perspectives. Trends Immunol 2015; 36: 217-228.

14. Freund A, Orjalo AV, Desprez PY, Campisi J. Inflammatory networks during cellular senescence: causes and consequences. Trends Mol Med 2010; 16: 238-246.

15. Laberge RM, Sun Y, Orjalo AV, Patil CK, Freund A, Zhou L et al. MTOR regulates the pro-tumorigenic senescence-associated secretory phenotype by promoting IL1A translation. Nat Cell Biol 2015; 17: 1049-1061.

16. Bhaumik D, Scott GK, Schokrpur S, Patil CK, Orjalo AV, Rodier F et al. MicroRNAs miR-146a/b negatively modulate the senescence-associated inflammatory mediators IL-6 and IL-8. Aging 2009; 1: 402-411.

17. Liu F, Wu S, Ren H, Gu J. Klotho suppresses RIG-I-mediated senescence-associated inflammation. Nat Cell Biol 2011; 13: 254-262.

18. Wang P, Han L, Shen H, Wang P, Lv C, Zhao G et al. Protein kinase D1 is essential for Ras-induced senescence and tumor suppression by regulating senescence-associated inflammation. Proc Natl Acad Sci USA 2014; 111: 7683-7688.

19. Kang $C, X u$ Q, Martin TD, Li MZ, Demaria M, Aron L et al. The DNA damage response induces inflammation and senescence by inhibiting autophagy of GATA4. Science 2015; 349: a5612.

20. Zhu H. Forkhead box transcription factors in embryonic heart development and congenital heart disease. Life Sci 2016; 144: 194-201.

21. Potter CS, Peterson RL, Barth JL, Pruett ND, Jacobs DF, Kern MJ et al. Evidence that the satin hair mutant gene Foxq1 is among multiple and functionally diverse regulatory targets for Hoxc13 during hair follicle differentiation. J Biol Chem 2006; 281: 29245-29255.

22. Verzi MP, Khan $\mathrm{AH}$, Ito $\mathrm{S}$, Shivdasani RA. Transcription factor Foxq1 controls mucin gene expression and grunule content in mouse stomach surface mucous cells. Gastroenterology 2008; 135: 591-600.

23. Kaneda H, Arao T, Tanaka K, Tamura D, Aomatsu K, Kudo K et al. FOXQ1 is overexpressed in colorectal cancer and enhances tumorigenicity and tumor growth. Cancer Res 2010; 70: 2053-2063. 
24. Zhang H, Meng F, Liu G, Zhang B, Zhu J, Wu F et al. Forkhead transcription factor foxq1 promotes epithelial-mesenchymal transition and breast cancer metastasis. Cancer Res 2011; 71: 1292-1301.

25. Qiao Y, Jiang X, Lee ST, Karuturi RK, Hooi SC, Yu Q. FOXQ1 regulates epithelialmesenchymal transition in human cancers. Cancer Res 2011; 71: 3076-3086.

26. Ross JB, Huh D, Noble LB, Tavazoie SF. Identification of molecular determinants of primary and metastatic tumour re-initiation in breast cancer. Nat Cell Biol 2015; 17: 651-664.

27. Huang W, Chen Z, Shang X, Tian D, Wang D, Wu K et al. Sox12, a direct target of FoxQ1, promotes hepatocellular carcinoma metastasis through up-regulating Twist1 and FGFBP1. Hepatology 2015; 61: 1920-1933.

28. Pei $Y$, Wang $P$, Liu H, He F, Ming L. FOXQ1 promotes esophageal cancer proliferation and metastasis by negatively modulating CDH1. Biomed Pharmacother 2015; 74: 89-94.

29. Feng J, Xu L, Ni S, Gu J, Zhu H, Wang $\mathrm{H}$ et al. Involvement of FoxQ1 in NSCLC through regulating EMT and increasing chemosensitivity. Oncotarget 2014; 5: 9689-9702.

30. Vishnubalaji R, Hamam R, Yue S, Al-Obeed O, Kassem M, Liu FF et al. MicroRNA-320 suppresses colorectal cancer by targeting SOX4, FOXM1, and FOXQ1. Oncotarget 2016; 7: 35789-35802

31. Xia L, Huang W, Tian D, Zhang L, Qi X, Chen Z et al. Forkhead box Q1 promotes hepatocellular carcinoma metastasis by transactivating ZEB2 and VersicanV1 expression. Hepatology 2014; 59: 958-973.

32. Kida Y, Goligorsky MS. Sirtuins, cell senescence, and vascular aging. Can J Cardiol 2016; 32: 634-641.

33. Vachharajani VT, Liu T, Wang X, Hoth JJ, Yoza BK, McCall CE. Sirtuins link inflammation and metabolism. J Immunol Res 2016; 2016: 8167273.
34. Kauppinen A, Suuronen T, Ojala J, Kaarniranta K, Salminen A. Antagonistic crosstalk between NF-kappaB and SIRT1 in the regulation of inflammation and metabolic disorders. Cell Signal 2013; 25: 1939-1948.

35. Cao X, Xue L, Han L, Ma L, Chen T, Tong T. WW domain-containing E3 ubiquitin protein ligase 1 (WWP1) delays cellular senescence by promoting p27(Kip1) degradation in human diploid fibroblasts. J Biol Chem 2011; 286: 33447-33456.

36. Serrano M, Lin AW, McCurrach ME, Beach D, Lowe SW. Oncogenic ras provokes premature cell senescence associated with accumulation of p53 and p16INK4a. Cell 1997; 88: 593-602.

(c) (i)

Cell Death and Disease is an open-access journal published by Nature Publishing Group. This work is licensed under a Creative Commons Attribution 4.0 International License. The images or other third party material in this article are included in the article's Creative Commons license, unless indicated otherwise in the credit line; if the material is not included under the Creative Commons license, users will need to obtain permission from the license holder to reproduce the material. To view a copy of this license, visit http://creativecommons.org/licenses/by/4.0/

(C) The Author(s) 2017

Supplementary Information accompanies this paper on Cell Death and Disease website (http://www.nature.com/cddis) 